\title{
Ueber das Schicksal cyklischer Terpene und Campher im thierischen Organismus. \\ (Dritte Mittheilung.)
}

\author{
Ueber das Verhalten des Camphens im Thierkörper. \\ Von
}

Emil Fromm-Freiburg i. B., Hermann Hildebrandt-Berlin und

Panl Clemens-Freiburg i. B.

(Aus der medicinischen Abtheilung des chemischen Universitätslaboratoriums zu Freiburg i. B. und

aus der chemischen Abtheilung des physiologischen Instituts zu Berlin.)

(Der Redaction zugegangen am 27. November 1902.)

In der ersten Mittheilung über das obige Thema ${ }^{1}$ ) haben Fromm und Hildebrandt gezeigt, dass nach der Verfütterung einer ganzen Reihe von Terpenen gepaarte Glycuronsäuren im Harn auftreten. Alle diese gepaarten Verbindungen können durch Kochen mit verdünnten Säuren in Glycuronsäure und ein sauerstoffhaltiges Derivat der betreffenden Terpene gespalten werden.

Die meisten dieser Oxyterpene zerfallen indessen bei der Spaltung selbst in Cymole und Wasser:

$$
\mathrm{C}_{10} \mathrm{H}_{18} \mathrm{O}=\mathrm{C}_{10} \mathrm{H}_{14}+\mathrm{H}_{2} \mathrm{O} \text {. }
$$

Unter allen untersuchten Terpenen ergab das Camphen allein im Thierkörper eine gepaarte Glycuronsäure, deren Spaltungsproduct gegen verdünnte Säuren beständig war. Hier ist es gelungen, aus dem Harn von Thieren, die mit Camphen gefüttert waren, ein flüchtiges Spaltungsproduct von der Formel $\mathrm{C}_{10} \mathrm{H}_{10} \mathrm{O} \mathrm{zu}$ isoliren, welches $\mathrm{Camphenol}$ genannt wurde. Die

1) Diese Zeitschrift, Bd. XXXIII, S. 579. 
Analysen dieser Substanz sind 1. c. mitgetheilt worden; weitere Versuche zur Ermittelung der Constitution dieser Verbindung wurden in Aussicht gestellt.

Wir haben nun grössere Mengen des sogenannten Camphenols dargestellt und dessen Constitution ermittelt, auch ist es uns in diesem Falle gelungen, die gepaarte Glycuronsäure aus dem Harn in Gestalt ihres Kalisalzes zu isoliren. Aus diesen Thatsachen lässt sich nun ein fast lückenloses Bild der chemischen Vorgänge bei der Verfütterung von Camphen im Thierkörper construiren, über welches wir im Folgenden berichten wollen.

Was zunächst die Constitution des sogenannten Camphenols anlangt, so haben wir in der ersten Mittheilung die Annahme gemacht, dass derselbe durch einfache Oxydation im Thierkörper entstünde und die Zusammensetzung eines Alkohols haben müsste:

$$
\mathrm{C}_{10} \mathrm{H}_{16}+\mathrm{O}=\mathrm{C}_{10} \mathrm{H}_{15} \mathrm{OH} \text {. }
$$

Dieser Alkohol wurde, so schien es, im Thierkörper an Glycuronsäure gepaart und ausgeschieden und aus der gepaarten Verbindung durch verdünnte Säure scheinbar unverändert wieder abgespalten. So einfach liegen indessen die Verhältnisse nicht. Als wir grössere Mengen des flüchtigen Spaltungsproductes (ca. $50 \mathrm{~g}$ ) zu unserer Verfügung hatten, zeigte sich allerdings zunächst wieder, dass weitaus der grösste Theil dieser Substanz wie früher bei $202-204^{\circ}$ überging und der Zusammensetzung $\mathrm{C}_{10} \mathrm{H}_{16} \mathrm{O}$ des sogenannten Camphenols entsprach. Nebenbei entstand indessen dieses Mal bei der Verarbeitung der grösseren Menge ein Nachlauf vom Siedepunkt ca. $260-270^{\circ}$, welcher krystallinisch erstarrte. Die Reinigung dieses Productes mit Hülfe von Holzgeist führte zu einer schön krystallisirten Verbindung $\mathrm{C}_{20} \mathrm{H}_{30} \mathrm{O}$, vom Schmelzpunkt $72^{\circ}$. Die geringen Mengen, in welchen diese Substanz entsteht, haben bisher eine Bestimmung ihrer Constitution unmöglich gemacht. $\mathrm{Da}$ indessen bei der häufig wiederholten Destillation des sogenannten Camphenols stets kleine Mengen von Wasser beobachtet wurden, so will es wohl scheinen, als sei diese neue Verbindung ein Kunstproduct, welches unter dem Einfluss er- 
höhter Temperatur durch Wasserabspaltung aus 2 Molekülen des sogenannten Camphenols entsteht.

$$
2 \mathrm{C}_{10} \mathrm{H}_{18} \mathrm{O}=\mathrm{C}_{20} \mathrm{H}_{30} \mathrm{O}+\mathrm{H}_{2} \mathrm{O} \text {. }
$$

Ein Theil der Fraction von 202-204 ${ }^{\circ}$ wurde $\mathrm{zu}$ einer Oxydation mit Permanganat in der Kälte verwendet. Diese Reaction, welche sehr leicht eintritt, hat uns gezeigt, dass der vermeintliche Camphenol überhaupt kein Alkohol, sondern sonderbarer Weise ein Aldehyd ist. Wir vermochten nämlich aus der alkalischen Oxydationsflüssigkeit eine Säure $\mathrm{C}_{10} \mathrm{H}_{16} \mathrm{O}_{2}$ $\mathrm{zu}$ isoliren. Die Reaction war also nach der Gleichung:

$$
\mathrm{C}_{10} \mathrm{H}_{16} \mathrm{O}+\mathrm{O}=\mathrm{C}_{10} \mathrm{H}_{16} \mathrm{O}_{2}
$$

verlaufen. Nach einer solchen Gleichung kann aber eine Säure nur aus einem Aldehyd entstehen. Einige Versuche erwiesen sofort die Richtigkeit unseres Schlusses. Der vermeintliche Camphenol reducirt Silberoxyd unter Spiegelbildung und liefert mit Disulfit eine prachtvoll krystallisirende Verbindung.

Die Säure $\mathrm{C}_{10} \mathrm{H}_{16} \mathrm{O}_{2}$, welche wir bei der oben erwähnten Oxydation erhalten haben, schmolz zunächst bei $102^{\circ}$, zeigte aber nach mehrfachem Umkrystallisiren aus verdünntem Alkohol den Schmelzpunkt $117-118^{\circ}$. Unter den in der Litteratur bekannten Verbindungen $\mathrm{C}_{10} \mathrm{H}_{16} \mathrm{O}_{2}$ zeigt nun Isocamphenilansäure von Bredt und Jagelki ${ }^{1}$ ) den Schmelzpunkt $118^{\circ}$. Da Isocamphenilansäure nach den genannten Forschern auch durch Oxydation mit Permanganat aus Camphenilanaldehyd zu erhalten ist, so konnte unsere Säure mit Isocamphenilansäure, unser sogenannter Camphenol mit Camphenilanaldehyd identisch sein. - Gegen die Identität des sogenannten Camphenols mit Camphenilanaldehyd sprach allein der Umstand, dass Camphenol bisher nur in flüssigem Zustande erhalten worden ist, während der Camphenilanaldehyd von Bredt und Jagelki als schön krystallisirende Verbindung vom Schmelzpunkt $68-70^{\circ}$ beschrieben wird. Wir haben deshalb den ganzen Rest unseres Camphenolvorraths durch Schütteln mit Disulfit in das krystallisirte Additionsproduct verwandelt und aus diesem, nach der Vorschrift von Bredt und Jagelki, durch Erwärmen mit

1) Lieb. Ann., Bd. 310, S. 127. 
Soda den Aldehyd wieder in Freiheit gesetzt. Das so gereinigte Product krystallisirte nun sofort, zeigte den verlangten Schmelzpunkt von $68-70^{\circ}$ und kreiste wie Camphenilanaldehyd auf dem Wasser. In Anbetracht seiner äusseren Eigenschaften, seines Schmelzpunktes, seiner Aldehydreactionen und seiner Ueberführbarkeit in Isocamphenilansäure können wir nun wohl nicht mehr umhin, unseren sogenannten Camphenol für identisch mit dem Camphenilanaldehyd von Bredt und Jagelki anzusehen.

Obwohl kaum ein Zweifel über diese Identität obwalten kann, wäre es dennoch erwünscht, unsere auf dem Umwege über den Thierkörper gewonnenen Präparate mit den auf rein chemischem Wege erzeugten zu vergleichen. Herr Professor Bredt hat die grosse Liebenswürdigkeit gehabt, uns zu versprechen, die fraglichen Präparate neu herzustellen, um uns den Vergleich in Zukunft zu ermöglichen.

$\mathrm{Ob}$ nun unser Spaltungsproduct wirklich endgültig identisch ist mit Camphenilanaldehyd oder nicht, so ist dasselbe doch jedenfalls kein Alkohol, sondern sicher ein Aldehyd $\mathrm{C}_{10} \mathrm{H}_{16} \mathrm{O}$. Demnach müssen wir wohl für unser Spaltungsproduct den Namen Camphenol wieder streichen. - Hiermit erhebt sich aber eine neue Frage. Es kann doch wohl der von uns abgespaltene Aldehyd nicht als solcher mit Glycuronsäure gepaart gewesen sein, um so weniger, als das Paarungsproduct Fehling'sche Lösung nicht direct reducirt, sondern erst nach seiner Spaltung durch verdünnte Säuren. Der durch Spaltung gewonnene Aldehyd muss also ein Abbau- oder Umwandlungsproduct desjenigen Körpers sein, welcher ursprünglich an Glycuronsäure gepaart war; letzterer aber muss an irgend einer Stelle seines Moleküls eine Hydroxylgruppe enthalten haben, mit deren Hülfe er sich mit Glycuronsäure verbunden hat.

Nach Bredt ${ }^{1}$ ) kommt dem Camphen die Formel I zu, Semmler ${ }^{2}$ ) stellt für diese Verbindung Formel II auf. Auch

1) Ber., Bd. 26, S. 3056.

2) Ber., Bd. 33, S. 3423 . 
die Formel III von Bredt und Jagelki ${ }^{1}$ ) für den Camphenilanaldehyd ist nicht unbestritten. ${ }^{2}$ )

I.<smiles>CC1(C)[C@H]2CC[C@@H]1C2</smiles>

II.<smiles>C=C1C2CCC1(C)C2(C)C</smiles>

III.<smiles>CC1(C)[C@@H]2CC[C@@H]1C2C=O</smiles>

Es lag nun nahe, anzunehmen, dass das Hydroxylderivat des Camphens, welches im Thierkörper erzeugt und an Glycuronsäure gepaart wird, der tautomeren Form des Camphenilanaldehyds entspricht, also das Derivat des Vinylalkohols IV sei.<smiles>CC1(C)[C@H]2CC[C@@]1(C)C2=CO</smiles>

Dieser Alkohol entspräche der Camphenformel II von Semmler, wäre aber in der Form IV nur so lange beständig, als er an Glycuronsäure gepaart ist, und würde sich im Moment seiner Abspaltung in die tautomere, beständigere Form des Camphenilanaldehyds III umlagern. Gegen diese Auffassung spricht die Untersuchung des Kalisalzes der gepaarten Verbindnng, wetches wir glücklicher Weise, wenn auch nur in geringer Menge, haben isoliren können. Paart sich der oben erwähnte Alkohol IV mit Glycuronsäure unter Austritt von Wasser, so entsteht eine gepaarte Glycuronsäure $\mathrm{C}_{16} \mathrm{H}_{24} \mathrm{O}_{7}$ :

$$
\mathrm{C}_{10} \mathrm{H}_{16} \mathrm{O}+\mathrm{C}_{6} \mathrm{H}_{10} \mathrm{O}_{7}=\mathrm{C}_{16} \mathrm{H}_{24} \mathrm{O}_{7}+\mathrm{H}_{2} \mathrm{O} \text {. }
$$

Dieser gepaarten Glycuronsäure müsste das Kalisalz $\mathrm{C}_{16} \mathrm{H}_{23} \mathrm{O}_{7} \mathrm{~K}$ entsprechen.

1) Lieb. Ann., Bd. 310, S. 116.

2) Chem. Zeitschrift (Ahrens), Bd. 1, S. 245. 
194 Emil Fromm, Hermann Hildebrandt, Paul Clemens.

Dieses Kalisalz haben wir analysenrein durch Umkrystallisiren aus kochendem absoluten Alkohol erhalten, indessen das mit Hülfe dieses Verfahrens gewonnene Product entsprach bei der Analyse der Formel $\mathrm{C}_{16} \mathrm{H}_{27} \mathrm{O}_{9} \mathrm{~K}$, enthielt also $2 \mathrm{H}_{2} \mathrm{O}$ mehr, als wir erwarten mussten. Wollten wir diese $2 \mathrm{H}_{2} \mathrm{O}$ lediglich als Krystallwasser betrachten, so wäre es immerhin schon auffallig, dass unser Salz sein Krystallwasser selbst bei der Behandlung mit kochendem absoluten Alkohol festhält. Deswegen haben wir versucht, ein wasserfreies Salz durch Trocknen bis zum constanten Gewicht herzustellen, und haben ermittelt, dass in der That beim Trorknen im Vacuum oder bei höschtens $110^{\circ}$ ein halbes Molekül Wasser verloren geht. Mehr Wasser gibt die Substanz unter keinen Umständen ab. Erst bei $140-150^{\circ}$ tritt ein erheblicherer Gewichtsverlust ein, welcher indessen erst nach mehreren Tagen eine Höhe erreicht, die dem Verlust von $2 \mathrm{H}_{2} \mathrm{O}$ entspricht. Bei einer solchen Trocknung bräunt sich die Substanz indessen erheblich und liefert einen Rückstand, dessen Analyse erkennen lässt, dass bei diesem Trocknen eher ein Verlust von Kohlenstoff als von Wasser eingetreten ist.

Wenn es uns nun bisher auch noch nicht gelungen ist, andere wohlkrystallisirende Salze, welche sich zu solchen Versuchen eignen, beizubringen, so dürfen wir nach diesem Resultat doch wohl die Meinung vertreten, dass von den 2 Molekülen Wasser des oben erwähnten Salzes höchstens eins als Krystallwasser, das andere aber sicher als Constitutionswasser aufzufassen ist.

Demnach haben wir es hier nicht mit einer Säure $\mathrm{C}_{16} \mathrm{H}_{24} \mathrm{O}_{7}$, sondern mit einer Säure $\mathrm{C}_{16} \mathrm{H}_{26} \mathrm{O}_{8}$ zu thun. Die Spaltung dieser Säure verläuft also in zwei Phasen, nämlich:

$$
\text { I. } \mathrm{C}_{16} \mathrm{H}_{28} \mathrm{O}_{8}+\mathrm{H}_{2} \mathrm{O}=\mathrm{C}_{10} \mathrm{H}_{18} \mathrm{O}_{2}+\mathrm{C}_{6} \mathrm{H}_{10} \mathrm{O}_{7} \text {; }
$$

nach dieser Gleichung zerfällt die gepaarte Säure in Glycuronsäure $\mathrm{C}_{6} \mathrm{H}_{10} \mathrm{O}_{7}$ und ein Derivat des Camphens $\mathrm{C}_{10} \mathrm{H}_{18} \mathrm{O}_{2}$. Letzteres ist unbeständig und zerfällt:

$$
\text { II. } \mathrm{C}_{10} \mathrm{H}_{18} \mathrm{O}_{2}=\mathrm{C}_{10} \mathrm{H}_{16} \mathrm{O}+\mathrm{H}_{2} \mathrm{O} \text {, }
$$

d. i. in Camphenilanaldehyd und Wasser. 
Das Camphen wird also im Thierkörper in erster Phase nicht bloss oxydirt, sondern oxydirt und hydratisirt. Die erste Gleichung lautet also:

$$
\mathrm{C}_{10} \mathrm{H}_{16}+\mathrm{O}+\mathrm{H}_{2} \mathrm{O}=\mathrm{C}_{10} \mathrm{H}_{18} \mathrm{O}_{2} \text {. }
$$

Die so erzeugte Verbindung $\mathrm{C}_{10} \mathrm{H}_{18} \mathrm{O}_{2}$ enthält wenigstens eine Hydroxylgruppe, mit welcher sie an Glycuronsäure gepaart wird.

Was die Natur des anderen Sauerstoffatomes anlangt, so kommt für dasselbe in Betracht, dass die im Thierkörper gebildete Verbindung $\mathrm{C}_{10} \mathrm{H}_{18} \mathrm{O}_{2}$ in freiem Zustande sehr leicht in Camphenilanaldehyd und Wasser zerfällt. Hierdurch wird natürlich der Gedanke nahe gelegt, dass der zweite Sauerstoff in der Verbindung $\mathrm{C}_{10} \mathrm{H}_{18} \mathrm{O}_{2}$ bereits als Aldehydsauerstoff existirt. Wäre dies der Fall, so müsste nicht nur die Verbindung $\mathrm{C}_{10} \mathrm{H}_{18} \mathrm{O}_{2}$, sondern auch die gepaarte Glycuronsäure eine freie Aldehydgruppe enthalten und demnach vor der Spaltung bereits Silberoxyd oder Fehling'sche Lösung reduciren. Dies ist aber, wie man sich leicht überzeugen kann, niemals der Fall, und somit ist diese Möglichkeit auszuschliessen.

Da nun aus leicht übersehbaren Gründen das zweite Sauerstoffatom der Verbindung $\mathrm{C}_{10} \mathrm{H}_{18} \mathrm{O}_{2}$ nicht wohl ätherartig gebunden sein kann, so bleibt nur die Möglichkeit übrig, dass dasselbe zur Bildung einer zweiten Hydroxylgruppe verwendet ist, dass also die Formel $\mathrm{C}_{10} \mathrm{H}_{19} \mathrm{O}_{2}$ in $\mathrm{C}_{10} \mathrm{H}_{16}(\mathrm{OH})_{2}$ aufzulösen ist.

Wir müssen demnach nach einem Dihydroxylderivat des Camphens fahnden, welches leicht Wasser verliert und dabei in Camphenilanaldehyd übergeht. Ein solches liegt nun bereits im Camphenglycol vor, welches Wagner ${ }^{1}$ ) durch vorsichtige Oxydation des Camphens erhalten und durch Erhitzen mit verdünnter Salzsäure in einen Aldehyd $\mathrm{C}_{10} \mathrm{H}_{16} \mathrm{O}$ verwandelt hat. Bredt ${ }^{2}$ ) vertritt die Ansicht, dass der Aldehyd, den Wagner auf diese Manier erhalten hat, mit Camphenilanaldehyd identisch ist, und dass die Umwandlung von Camphenglycol in Camphenilanaldehyd analog der Umwandlung von Hydrobenzoin im Diphenylacetaldehyd nach folgender Gleichung verlaufe:

1) Ber., Bd. 23, S. 2309.

2) Lieb. Ann., Bd. 310, S. 118. 


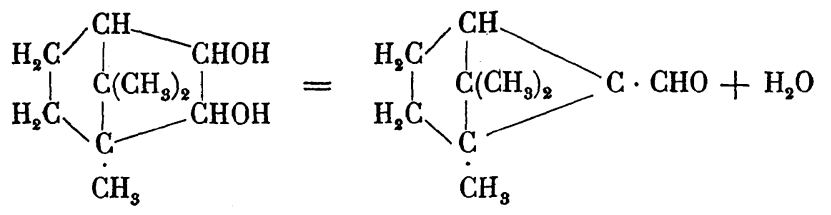

Wir dürfen nun wohl unsere Betrachtungen dahin zusammenfassen, dass Camphen im Thierkörper höchst wahrscheinlich in Camphenglycol verwandelt wird, und dass der Glycol gleichfalls durch den Organismus mit einer seiner Hydroxylgruppen an Glycuronsäure gepaart wird. Es erscheint also demnach das Camphen im Harn nicht als Camphenolglycuronsäure, wie wir früher angenommen haben, sondern als Camphenglycolmonoglycuronsäure. Bemerkenswerth ist, dass der Thierkörper von zwei ihm gebotenen Hydroxylgruppen nur eine benutzt, um an dieselbe Glycuronsäure zu paaren; bemerkenswerth ist ferner, dass der Thierkörper das Camphen, ebenso wie den Campher, an derselben Stelle oxydirend angreift, an welcher es auch von anorganischen Oxydationsmitteln angegriffen wird.

Bei allen diesen Betrachtungen darf indessen ein Umstand nicht übersehen werden, nämlich der, dass das camphenglycolmonoglycuronsaure Kali stets nur in verschwindend kleiner Menge erhalten wurde. Die alkoholischen Mutterlaugen von dieser Substanz enhalten ungleich viel grössere Mengen von Kalisalzen gepaarter Glycuronsäuren, welche auf keine Weise zum Krystallisiren zu bringen gewesen sind. Die nicht krystallisirenden Salze aus den Mutterlaugen reduciren nach dem Kochen mit verdünnten Säuren sehr stark und liefern bei der Spaltung reiche Ausbeuten von Camphenilanaldehyd. Diese Salze stehen also zweifellos dem camphenglycolmonoglycuronsauren Kali sehr nahe; ob sie mit diesem Salz identisch sind und nur durch irgendwelche Verunreinigung am Krystallisiren verhindert werden, ob sie sich vielleicht von dem krystallisirenden Salz durch höheren oder geringeren Gehalt von Wasser unterscheiden, oder ob sie endlich gar vielleicht die Diglycuronsäureverbindungen des Camphenglycols sind, haben wir nicht entscheiden können, da es uns nicht gelungen 


\section{Druckfehler.}

\section{Band XXXVII, Seite 196 ist}

statt:

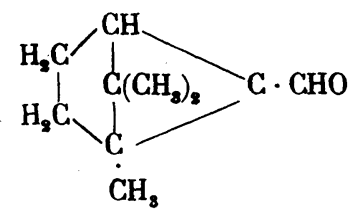

zu lesen:

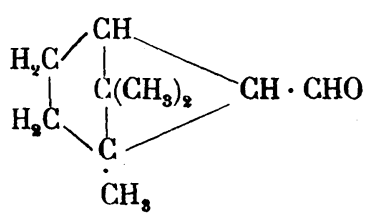


ist, die nicht unerheblichen Quantitäten dieser Verbindungen in analysenreine Form zu bringen.

\section{Beschreibung der Versuche.}

Das zu unseren Versuchen verwendete d-Camphen verdanken wir der Güte der Firma Schimmel \& Co., Leipzig-Miltitz, welcher wir auch an dieser Stelle für ihr freundliches Entgegenkommen unseren Dank sagen. Bei Zusatz von 1/3 Volumen Olivenöl bleibt das Camphen auch bei Zimmertemperatur flüssig. $\mathrm{Zu}$ den Versuchen wurden Kaninchen verwendet, welchen die Lösung von Camphen in Oel mittelst der Schlundsonde beigebracht wurde.

Der bei diesen Versuchen gewonnene Harn wird, so frisch als möglich, mit neutralem Bleiacetat versetzt; der dabei entstehende Niederschlag enthält die gesuchten Substanzen nicht und wird durch Abfiltriren beseitigt. Aus dem Filtrat wird durch basisches Bleiacetat ein reichlicher Niederschlag gewonnen, welcher die gesuchte Substanz enthält, auf dem Filter gesammelt, gewaschen und als Rohmaterial verwendet wird. Dieses Ausgangsmaterial werden wir im Folgenden kurzweg als «Bleiniederschlag» bezeichnen.

\section{Gewinnung von Camphenilanaldehyd.}

Der «Bleiniederschlag» wird mit verdünnter Schwefelsäure angerieben, zum Sieden erhitzt und mit Wasserdampf destillirt. Das auf dem Destillat schwimmende Oel haben wir abgehoben, getrocknet und destillirt. Hierbei ergab sich neben einem geringen Vorlauf im Wesentlichen flüssiger Camphenilanaldehyd, dessen Analyse bereits früher (l. c.) mitgetheilt wurde, vom Siedepunkt 202-2040, und ein Nachlauf vom Siedepunkt $260-270^{\circ}$, von welchem unten die Rede sein wird. Beim Erhitzen einiger Tropfen des Productes vom Siedepunkt $202-204^{\circ}$ mit einer Auflösung von Silbernitrat in Ammoniak bildet sich sofort ein schöner Silberspiegel. Wurde das Product vom Siedepunkt 202-2040 mit concentrirter Natriumdisulfitlösung und einigen Tropfen Alkohol geschüttelt, so bildeten sich nach kurzer Zeit reichliche Mengen von 
Krystallen der Natriumdisulfitverbindung des Camphenilanaldehyds. Diese Krystalle wurden auf dem Saugfilter gesammelt, mit wenig verdünntem Alkohol gewaschen und dann kurze Zeit mit Sodalösung erhitzt; der erkalteten Lösung entzieht nun Aether reinen Camphenilanaldehyd, welcher vom verdunstenden Aether in Gestalt eines rasch erstarrenden Oeles zurückgelassen wird. Die so gewonnenen Krystalle schmelzen bei $68-70^{\circ}$, riechen angenehm und campherartig und kreisen ähnlich dem Campher, wenn sie auf Wasser geworfen werden. Schon bei Zimmertemperatur ist übrigens die Substanz zum Theil flüchtig und sublimirt in schönen Krystallen, was man besonders schön in flachen, mit Uhrgläsern bedeckten Gefässen beobachten kann. Dieselbe Substanz haben wir übrigens aus absolut reinem camphenglycolmonoglycuronsaurem Kali, sowie aus dem nicht ganz reinen Baryumsalz durch Spaltung mit Schwefelsäure gewonnen.

\section{Verbindung $\mathrm{C}_{20} \mathrm{H}_{30} \mathrm{O}$.}

Bei der Destillation des rohen Camphenilanaldehyds ergibt sich, wie oben erwähnt, ein Nachlauf vom Siedepunkt ca. 260-270 . Bei längerem Stehen erstarrt dieser Nachlauf grösstentheils krystallinisch. Die Krystalle werden durch Absaugen und Aufstreichen auf Thonteller von anhaftendem Oele befreit und in wenig Methylalkohol gelöst. Durch starke Abkühlung der methylalkoholischen Lösung mit Eiswasser erhält man so schöne Nadeln vom Schmelzpunkt $72^{\circ}$, welche der Formel $\mathrm{C}_{20} \mathrm{H}_{30} \mathrm{O}$ entsprechen.

$0,1828 \mathrm{~g}$ Substanz gaben $0,5587 \mathrm{~g} \mathrm{CO}_{\mathbf{z}}$, entsprechend $83,36 \% \mathrm{C}$, und $0,17 \mathrm{~g} \mathrm{H}_{2} \mathrm{O}$, entsprechend $10,28 \% \mathrm{H}$.

$\begin{array}{rlrl} & \text { Berechnet für } \mathrm{C}_{\mathbf{2 0}} \mathrm{H}_{30} \mathrm{O}: & \text { Gefunden: } \\ \mathrm{C}_{20}=240 & 83,91 \% & 83,36 \% \\ \mathrm{H}_{30}=30 & 10,49 \% & 10,49 \% \\ 0=\frac{16}{286} & & \end{array}$

\section{Isocamphenilansäure.}

Versetzt man den flüssigen oder festen Camphenilanaldehyd mit Kaliumpermanganat, so tritt augenblicklich Oxydation ein. 
Um das Oxydationsproduct zu gewinnen, haben wir mit 5\% iger Permanganatlösung versetzt und geschüttelt, solange als noch Permanganat verbraucht wurde. Hierbei wurde durch gelegentliches Eintauchen in kaltes Wasser dafür gesorgt, dass die Temperatur der Flüssigkeit nicht wesentlich über die des Zimmers stieg. Nach Beendigung der Oxydation haben wir mit einigen Tropfen Alkohol entfärbt, die alkalische Flüssigkeit vom Braunstein abgesaugt, eingeengt und mit Schwefelsäure angesäuert. Hierbei schied sich sofort eine feste Säure $a b$, welche der Flüssigkeit durch Ausschütteln mit Aether entzogen und durch Verdunsten des Aethers gewonnen wird. Aus Wasser, unter Zusatz einiger Tropfen Alkohol umkrystallisirt, zeigte die Säure den Schmelzpunkt $102^{\circ}$ und entsprach der Formel $\mathrm{C}_{10} \mathrm{H}_{16} \mathrm{O}_{2}$.

$0,1532 \mathrm{~g}$ Substanz gaben 0,4012 $\mathrm{g} \mathrm{CO}_{2}$, entsprechend $71,41 \% \mathrm{C}$, und $0,1275 \mathrm{~g} \mathrm{H}_{2} \mathrm{O}$, entsprechend $9,24 \% \mathrm{H}$.

$\begin{array}{rcc} & \text { Berechnet für } \mathrm{C}_{10} \mathrm{H}_{16} \mathrm{O}_{2}: & \text { Gefunden: } \\ \mathrm{C}_{10}=120 & 71,42 \% & 71,41 \% \\ \mathrm{H}_{16}=16 & 9,52 \% & 9,24 \% \\ \mathrm{O}_{2}=\frac{32}{168} & & \end{array}$

Da auf Grund dieser Analyse dem Oxydationsproduct die Formel einer Säure $\mathrm{C}_{10} \mathrm{H}_{16} \mathrm{O}_{2}$ zugewiesen werden musste, und da ferner unter den in der Litteratur bekannten Säuren dieser Formel die Isocamphenilansäure von Bredt und Jagelki vom Schmelzpunkt $118^{\circ}$ beschrieben war, so lag die Möglichkeit nahe, dass unsere Säure mit der von Bredt und Jagelki beschriebenen identisch wäre. Wir haben demnach, der Angabe jener Autoren folgend, unsere Säure aus Ligroin umkrystallisirt und in der That beobachtet, dass der Schmelzpunkt auf $117-1.18^{\circ}$ stieg, trotzdem uns nur wenig Material zur Verfügung stand. Wie Bredt's Isocamphenilansäure geht auch unsere Säure mit Wasserdämpfen leicht über, auch Bredt und Jagelki haben ihre Säure durch Permanganatoxydation aus dem Aldehyd erhalten.

Zum Ueberfluss haben wir die gereinigte Säure vom Schmelzpunkt $117-118^{\circ}$ noch einmal analysirt und auch hier die erwarteten Werthe gefunden, so dass gar kein Zweifel 
mehr obwalten kann, dass unsere Säure mit Isocamphenilansäure identisch ist.

0,1953 g Substanz ergaben 0,5094 $\mathrm{g} \mathrm{CO}_{2}$, entsprechend $71,14 \% \mathrm{C}$, und $0,1740 \mathrm{~g} \mathrm{H}_{2} \mathrm{O}$, entsprechend $9,88 \% \mathrm{H}$.

\begin{tabular}{ccc} 
& \multicolumn{3}{c}{$0,1740 \mathrm{~g} \mathrm{H}_{2} \mathrm{O}$, entsprechend } & $9,88 \%$ & $\mathrm{H}$ \\
& Berechnet für $\mathrm{C}_{10} \mathrm{H}_{16} \mathrm{O}_{\mathbf{2}}:$ & Gefunden: \\
$\mathrm{C}_{10}=120$ & $71,42 \%$ & $71,14 \%$ \\
$\mathrm{H}_{16}=16$ & $9,52 \%$ & $9,88 \%$ \\
$\mathrm{O}_{2}=\frac{32}{168}$ & &
\end{tabular}

Camphenglycolmonoglycuronsaures Kali.

Zur Gewinnung dieses Salzes wurde in einigen Fällen der «Bleiniederschlag », nach sorgfältigem Auswaschen in Wasser, suspendirt und mit Schwefelwasserstoff vom Blei befreit. Das saure Filtrat wurde mit Kalilauge neutralisirt, vorsichtig eingeengt und schliesslich im Vacuum völlig getrocknet. Bei anderen Darstellungen haben wir den "Bleiniederschlag» nach dem Auswaschen mit überschüssigem Kaliumsulfid angerührt und nach eingetretener Reaction das überschüssige Alkali mit Schwefelsäure neutralisirt. Nun wurde filtrirt, im Vacuum eingedampft und die concentrirte Lösung mit etwas Alkohol versetzt. Hierdurch fiel der grösste Theil des Kaliumsulfats aus. Man filtrirte ab, engte, wie oben, vorsichtig. ein und trocknete schliesslich im Vacuum völlig. Die zähe vacuumtrockene Masse wird durch Absaugen von anorganischen Bestandtheilen, die in derselben suspendirt sind, getrennt. Dem Rückstand entzieht siedender absoluter Alkohol eine Substanz, welche aus der erkaltenden Flüssigkeit krystallinisch wieder ausfällt und welche die in der Ueberschrift genannte Verbindung darstellt. Auf dem Filter gesammelt und im Vaccum getrocknet, erwies sich diese Substanz als kalihaltig, reducirte Fehling'sche Lösung nicht, lieferte beim Kochen mit verdünnten Säuren Camphenilanaldehyd und eine Fehling'sche Lösung reducirende Substanz, nämlich Glycuronsäure. Die Lösung der unversehrten Substanz drehte die Polarisationsebene nach links. Die Substanz löst sich ausserordentlich leicht in Wasser, krystallisirt aber aus dieser Lösung nicht mehr, sondern trocknet im Vacuum zu einer klebrigen, amorphen Masse ein. 
Exsiccatortrocken zeigte die Substanz bei der Analyse Werthe, welche einer Formel $\mathrm{C}_{16} \mathrm{H}_{23} \mathrm{O}_{7} \mathrm{~K}+2 \mathrm{H}_{2} \mathrm{O}$ entsprechen. $0,206 \mathrm{~g}$ Substanz gaben 0,355 $\mathrm{g} \mathrm{CO}_{2}$, entsprechend $48,09 \% \mathrm{C}$, und $0,1265 \mathrm{~g} \mathrm{H}_{2} \mathrm{O}$, entsprechend $6,82 \% \mathrm{H}$. $0,243 \mathrm{~g}$ Substanz gaben $0,0495 \mathrm{~g} \mathrm{SO}_{4} \mathrm{~K}_{2}$, entsprechend $9,12 \% \mathrm{~K}$.

$$
\begin{array}{ccc} 
& \text { Berechnet für } \mathrm{C}_{16} \mathrm{H}_{23} \mathrm{O}_{7} \mathrm{~K}+2 \mathrm{H}_{2} \mathrm{O}: & \text { Gefunden: } \\
\mathrm{C}_{16}=192 & 47,76 \% & 48,09 \% \\
\mathrm{H}_{27}=27 & 6,71 \% & 6,82 \% \\
\mathrm{O}_{9}=144 & - & - \\
\mathrm{K}=\frac{39}{402} & 9,70 \% & 9,12 \%
\end{array}
$$

Im Vacuum über Schwefelsäure bis zum constanten Gewicht getrocknet, zeigte das Kalisalz Werthe, welche einer Formel $\mathrm{C}_{16} \mathrm{H}_{23} \mathrm{O}_{7} \mathrm{~K}+1^{1 / 2} \mathrm{H}_{2} \mathrm{O}$ entsprechen. In diesem wie im folgenden Falle wurde das Salz im Schiffchen verbrannt und die Potasche im Schiffchen gewogen, so dass das Resultat der Verbrennung gleichzeitig zur Kalibestimmung verwendet werden konnte.

$0,1813 \mathrm{~g}$ Substanz hinterliessen im Schiffchen $0,0301 \mathrm{~g} \mathrm{~K}_{\mathbf{2}} \mathrm{CO}_{3}$,
entsprechend $9,38 \% \mathrm{~K}$ und $1,43 \% \mathrm{C}$,
und ergaben $0,3154 \mathrm{~g} \mathrm{CO} \mathrm{O}_{2}$, entsprechend $47,44 \% \mathrm{C}$, sowie $0,1098 \mathrm{~g} \mathrm{H}_{2} \mathrm{O}$, entsprechend $6,72 \% \mathrm{H}$.

$$
\begin{array}{lcc} 
& \text { Berechnet für } \mathrm{C}_{16} \mathrm{H}_{25} \mathrm{O}_{7} \mathrm{~K}+1^{\sharp / 2} \mathrm{H}_{8} \mathrm{O}: & \text { Gefunden: } \\
\mathrm{C}_{16}=192 & 48,85 \% & 48,87 \% \\
\mathrm{H}_{88}=26 & 6,62 \% & 6,72 \% \\
\mathrm{O}^{11_{2}}=136 & - & - \\
\mathrm{K}=\frac{39}{393} & 9,92 \% & 9,38 \%
\end{array}
$$

Wurde das Salz bei $105-110^{\circ}$ getrocknet, so verlor dasselbe nicht mehr an Gewicht, erst bei einer Temperatur von $140-150^{\circ}$ trat ein erheblicherer Gewichtsverlust ein.

$0,2228 \mathrm{~g}$ Substanz verloren bei mehrtägigem Trocknen bei $140-150^{\circ} 0,0198 \mathrm{~g}$ an Gewicht, was einem Verlust von $8,8 \%$ entpricht. Obwohl Gewichtsconstanz noch keineswegs erreicht war, wurde hier mit dem Trocknen aufgehört, einmal, weil sich die Substanz deutlich gebräunt hatte, und dann, weil sich für einen Verlust von $2 \mathrm{H}_{2} \mathrm{O} 8,9 \%$ berechnen. Ein Theil der so getrøckneten Substanz wurde analysirt. 
$0,1563 \mathrm{~g}$ Substanz hinterliessen im Schiffchen 0,0307 $\mathrm{g} \mathrm{K}_{2} \mathrm{CO}_{3}$, entsprechend $11,5 \% \mathrm{~K}$ und $1,7 \% \mathrm{C}$,

und gaben $0,2635 \mathrm{~g} \mathrm{CO}_{2}$, entprechend 45,97\% C, und 0,0927 $\mathrm{g} \mathrm{H}_{2} \mathrm{O}$, entsprechend $6,59 \% \mathrm{H}$.

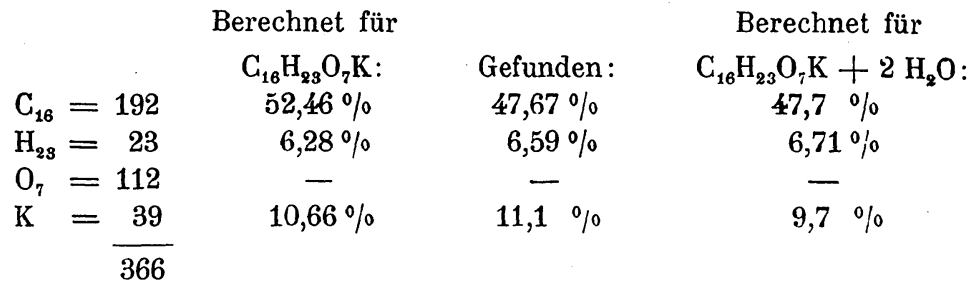

Ueberblickt man die obigen Analysenresultate, so ergibt sich zweifellos, dass unser Kalisalz durch das Trocknen bei $150^{\circ}$ eine Zersetzung unter Verlust von Kohlenstoff erlitten hat. Während der Procentgehalt an Kalium über die für wasserfreies Salz verlangte Zahl gestiegen ist, ist der Procentgehalt an Kohlenstoff weit hinter dem für diese Verbindung verlangten Werth zurückgeblieben. Es ist zweifellos ein $\mathrm{Zu}-$ fall, dass Kohlenstoff- und Wasserstoffgehalt der bei $150^{\circ}$ getrockneten Suhstanz, welche doch beinahe $9 \%$ an Gewicht verloren hat, immer noch mit dem Kohlenstoff- und Wasserstoffgehalt der Verbindung $\mathrm{C}_{16} \mathrm{H}_{23} \mathrm{O}_{7} \mathrm{~K}+2 \mathrm{H}_{2} \mathrm{O}$ übereinstimmen; dass der Rückstand dieser Verbindung nicht entspricht, geht absolut klar aus dem Kaligehalt hervor, welcher den verlangten Werth um fast $11 / 2 \%$ übersteigt.

Man konnte hoffen, durch Oxydation des camphenglycolmonoglycuronsauren Kalis zu Abbauproducten zu gelangen, welche Schlüsse auf die Constitution des Ausgangsmaterials ermöglicht hätten. In der That erhält man auch durch Oxydation des reinen Kalisalzes wie auch seiner nicht krystallisirenden Nebenproducte kleine Mengen krystallisirender Säuren, deren Schmelzpunkte bis gegen $145^{\circ}$ steigen. Diese Säuren sind aber Gemenge und die Ausbeuten an denselben sind so verschwindend kleine, dass es uns nicht möglich war, zu analysenreinen Producten zu gelangen. 